\title{
MULTISCROLL IN COUPLED DOUBLE SCROLL TYPE OSCILLATORS
}

\author{
SYAMAL KUMAR DANA \\ Central Instrumentation, Indian Institute of Chemical Biology, Jadavpur, Kolkata 700032, India \\ skdana@iicb.res.in \\ BRAJENDRA K. SINGH \\ Department of Infectious Disease Epidemiology, Imperial College London, St Mary's Campus, London W2 1PG, UK \\ b.singh@imperial.ac.uk
}

\author{
SATYABRATA.CHAKRABORTY1 ${ }^{1}$ RAM CHANDRA YADAV ${ }^{2}$ \\ Central Instrumentation, Indian Institute of Chemical Biology, Jadavpur, Kolkata 700032, India \\ ${ }^{1}$ satya_iicb@yahoo.com, ${ }^{2}$ rcyadav@iicb.res.in \\ JÜRGEN KURTHS \\ Institute of Physics, University of Potsdam, Am Neuen Palais, D-14415 Potsdam, Germany \\ juergen@agnld.uni-potsdam.de \\ GREGORY V. OSIPOV \\ Faculty of Computational Mathematics and Cybernetics, University of Nizhny Novgorod, \\ 603950 Nizhny Novogorod, Russia \\ osipov@rf.unn.ru
}

PRODYOT KUMAR ROY

Department of Physics, Presidency College, Kolkata 700073, India

pkpresi@yahoo.co.in

CHIN-KUN HU

Institute of Physics, Academia Sinica, Nankang, Taipei 11529, Taiwan and Center for Nonlinear and Complex Systems and Department of Physics, Chung-Yuan Christian University, Chungli 32023, Taiwan

huck@phys.sinica.edu.tw

\begin{abstract}
A unidirectional coupling scheme is investigated in double scroll type chaotic oscillators that reveal interesting multiscroll dynamics. Instead of using self-oscillatory systems, in this scheme, double scroll chaos from one oscillator is forced into another similar oscillator in a resting state. This coupling scheme is explored in the Chua oscillator, a modified Chua oscillator and the Lorenz oscillator. We have modified the Chua oscillator by simply changing its piecewise linear function a little bit and thereby derived a new 3-scroll attractor. We have observed 4-scroll, 6-scroll attractors in the driven Chua oscillator and the modified Chua oscillator respectively in an intermittency regime of weaker coupling. We have extended the coupling scheme to the Lorenz system when even more interesting multiscroll dynamics (3-, 4-, 5-, 6-scroll) is observed with decreasing coupling strength. It appears as if a hidden multiscroll structure unfolds with weakening coupling interactions. One after another additional scroll appears in the driven Lorenz system when the coupling strength is gradually decreased in the weaker coupling regime. The origin of such multiscroll dynamics is explained using eigenvalue analysis and a bifurcation diagram. A schematic diagram of the multiscroll trajectories is presented to further elucidate the evolution of the scrolls. Experimental evidences are also presented using the Chua circuit and an electronic analog of the Lorenz system.
\end{abstract}

\section{Introduction}

Studies on multiscroll chaos are made [Lü and Chen, 2006 and refs. therein], in recent times, in search of suitable hyperchaotic circuits for applications in secure communication and in other chaos-based information technologies. The basis of such multiscroll dynamics is mostly double scroll systems like the Chua oscillator [Chua, Komuro and Matsumoto, 1986]. The Chua oscillator has a piecewise linear function with three linear parts and two breakpoints which is an essential criterion for double scroll dynamics. The trajectory of double scroll in the Chua oscillator vtates around either of two saddle foci symmetric to a saddle focus at the origin. The trajectory switches irregularly from one to the other symmetric locations but always repelled by the third saddle focus origin. Multiscroll attractors have a larger number of scrolls ( $n>2$, $n$ is the number of scroll) and mostly been implemented [Kapitaniak et al, 1990; Kapitaniak et al, 1994; Suykens et al, 1993; Arena et al, 1996; Tang et al, 2001; Yalcin et al, 2000; Yalcin et al, 2001; Lü et al, 2003; Ozoguz et al, 2002; Cafagna et al, 2003; Lii et al, 2004; Lü et al, 2004a; Yu et al, 2006; Lii et al, 2006] by introducing additional saddle foci in terms of added breakpoints in the model system. Multiscroll has been reported in unidirectionally or diffusively coupled self-oscillating Chua circuits [Kapitaniak and 
Chua, 1994] and in arrays of 1-D cellular neural networks (CNN) [Suykens and Vandewalle, 1993], where the birth of a double-double scroll attractor is found in intermittent bursts from 3D manifold to higher dimensions for weaker coupling. However, in such attempts too, for the generation of multiscroll, additional break point was necessitated in the piecewise linear function of the Chua circuit. The electronic implementation of multiscroll is done with different circuit schemes by adding multiple breakpoints in the piecewise linear function of the system [Kapitaniak et al, 1994; Suykens et al, 1993; Arena et al, 1996; Suykens et al, 1997; Yalcin et al, 2000; Yalcin et al, 2001, Cafagna et al, 2003], by using a sine function [Tang et al, 2001] or by adding saturated function series [Lü et al, 2004]. Hysteresis [Lü et al, 2004a] is also used sometimes for the generation of $\mathrm{n}$-scroll chaos. The important practical applications of multiscroll are found as broadband signal generator and as true pseudorandom number generator for communication engineering.

The Lorenz system is another class of double scroll system where the nonlinearity exists not in the form of any piecewise linear function but in a straightforward manner in the governing system equations. The trajectory of a typical butterfly attractor in the Lorenz system rotates most of the time around either of its two symmetric saddle foci and always repelled by the saddle origin. Some attempts [Lü and Chen, 2006, Yu et al, 2006] have been recently made to generate multiscroll using the Lorenz system. A general multiscroll Lorenz system family has been recently introduced [Yu et al, 2006] by using a nonparametric polynomial transformation in the Lorenz system. A self-feedback control algorithm[Singh and Hu, 2005] has also been found to work nicely to generate multiscroll in a single Lorenz system.

In this paper, our main motivation is to investigate the interaction of a chaotic oscillator with another similar oscillator in a resting state using double scroll systems like the Chua oscillator and the Lorenz system and thereby to explore the multiscroll dynamics. In most of the earlier studies of multiscroll dynamics in coupled systems and also on synchronization studies [Osipov, Kurths and Zhou, 2007; Pikovsky, Rosenblum and Kurths, 2000, 2001; Pecora and Carroll, 1995], in general, the major thrust was given on two or more interacting self-oscillating systems. Instead, we stress here on two coupled nonlinear systems where one is oscillatory and intercepting unidirectionally another system in a resting state. However, similar to earlier results [Kapitaniak and Chua, 1994; Suykens and Chua, 1997] in self-oscillating coupled systems, we observed 2n-scroll ( $\mathrm{n}=2-$, 3 -scroll) chaos in intermittent bursts in both double scroll $(n=2)$ Chua oscillator and 3-scroll $(n=3)$ modified Chua oscillator. Our objective is to show how an $n$-scroll $(n=2,3, \ldots)$ system can generate a $2 n$-scroll attractor when an oscillatory system interact with a resting system via unidirectional coupling. We simulate a new 3 -scroll system for this purpose. In fact, a 3 -scroll attractor is reported earlier using either a sine function [Tang et al, 2001] or a voltage controlled current source [Yalcin, Suykens and Vandawalle, 2000] in the Chua circuit. Instead, we derive the new 3-scroll attractor simply by making a small change in the piecewise linear function of the Chua oscillator. Next, we extend the unidirectional coupling scheme to Lorenz system and are able to generate interesting multiscroll dynamics as if a complex hidden geometry gradually unfolds with decreasing coupling interaction. One after another scroll (3-, 4, 5-, 6-scroll) is added to the driven Lorenz system when the coupling interaction is decreased. We confirm our numerical results with electronic experiments using the Chua circuit and an analog Lorenz circuit.

The paper is structured as follows. In section 2, we present the numerical results of the multiscroll dynamics using Chua oscillator, modified Chua oscillator and present the electronic experiment using two coupled Chua circuit. In section 3, we describe our numerical and experimental results in coupled Lorenz system. We explained the origin of multiscroll in the coupled Lorenz system by using eigenvalue analysis and by revealing the bifurcation scenario. We present a schematic diagram of the multiscroll trajectories in coupled Lorenz system to elucidate their evolution in the context of stability of the equilibrium points. Finally we summarize the results in section 4.

\section{Multiscroll in coupled Chua's Circuit}

Coupled systems are usually investigated from the viewpoint of synchronization [Osipov, Kurths and Zhou, 2007; Pikovsky, Rosenblum and Kurths, 2001] which involves two or more self-oscillatory systems. In exploring multiscroll dynamics too, self-oscillatory chaotic systems are studied using drive-response type unidirectional coupling in the Chua circuit [Kapitaniak and Chua, 1994] or CNNs [Suykens and Chua, 1997] with added breakpoints. An intermittency regime is reported in such coupled self-oscillatory double scroll systems. In this intermittency regime of coupled double scroll or 2 scroll oscillators, a 4-scroll attractor is really born when the double scroll attractor shows intermittent jumps to another double scroll in higher dimension. Instead of using two self-oscillatory systems, here we use two oscillators, one in an oscillatory state and the other one in a resting state. In other words, the double scroll chaos from one Chua oscillator is forced into the other Chua oscillator in a resting state. Nevertheless, we found [Roy, Chakraborty and Dana, 2003] a similar intermittency regime where the 4-scroll attractor is seen again. No additional breakpoint is necessitated that reduces the circuit complexity. Both numerical as well as experimental results are presented to show how a 4-scroll is obtained. We extend the results to generate 6-scroll attractor using coupled 3-scroll system. The 3-scroll system is simulated by simply modifying the inner slope of the piecewise nonlinear function of the original Chua circuit. 


\subsection{Numerical Simulation: Coupled Chua Oscillator}

The Chua oscillator is well known for its double scroll chaos [Chua, Komuro and Matsumoto, 1986; Kennedy, 1993; Dana, Blasius and Kurths, 2006]. The oscillator has three saddle foci, one at the origin (eigenvalues: $\gamma_{0},-\sigma_{0} \pm j \omega_{0}$ ) and two more at mirror symmetric positions (eigenvalues: $-\gamma_{1}, \sigma_{1} \pm j \omega_{1}$ ) to the origin. The trajectory of the double scroll attractor rotates around either of the two mirror symmetric saddle foci and switches irregularly between themselves. The trajectory is always repelled by the saddle origin. The governing equations of the unidirectionally coupled Chua oscillator, in dimensionless form, are given by

$$
\begin{aligned}
& \frac{d x_{1}}{d t}=\alpha_{1}\left[y_{1}-x_{1}-f\left(x_{1}\right)\right] \quad \text { (1a) } \frac{d x_{2}}{d t}=\alpha_{2}\left[y_{2}-x_{2}-f\left(x_{2}\right)\right]+\varepsilon\left(x_{1}-x_{2}\right) \\
& \frac{d y_{1}}{d t}=x_{1}-y_{1}+z_{1} \\
& \frac{d z_{1}}{d t}=-\beta_{1} y_{1}-\gamma_{1} z_{1} \\
& \frac{d z_{2}}{d t}=-\beta_{2} y_{2}-\gamma_{2} z_{2}
\end{aligned}
$$

where the piecewise linear function $f(\bullet)$, in a general form, is expressed by

$$
f\left(x_{1,2}\right)= \begin{cases}b_{1,2} x_{1,2}+\left(b_{1,2}-a_{1,2}\right) & \text { if } x_{1,2}<-1 \\ a_{1,2} x_{1,2} & \text { if }-1 \leq x_{1,2} \leq 1 \\ b_{1,2} x_{1,2}+\left(a_{1,2}-b_{1,2}\right) & \text { if } x_{1,2}>1\end{cases}
$$

Subscripts 1, 2 denote the driver and the response oscillator respectively, i.e., the equations 1(a)-(c) represent the driver and the equations 1(d)-(f) for the response oscillator. The term $\varepsilon\left(x_{1}-x_{2}\right)$ in eq.(1d) denotes the unidirectional coupling where the coupling is made via the $\mathrm{x}$-coordinate only and $\varepsilon$ is the coupling strength. The system parameters $\alpha_{i}, \beta_{i}$ and $\gamma_{i}(i=1,2)$ are so chosen (details given in the circuit diagram in Fig.1) that the driver is double scroll chaotic and the response is in a resting state when uncoupled. For strong coupling, the response oscillator is in almost complete synchrony with the driver for a coupling strength above a threshold, $\varepsilon=\varepsilon_{C}$ when both amplitude and phase of the driver and the response are almost identical. In numerical simulations, we find this coupling threshold as $\varepsilon_{C}=10.5$ for the selected parameter regime. If the coupling strength is then gradually decreased, a 4-scroll attractor appears in the response oscillator as shown in 3D plot of Fig.1(a) for a range of weaker coupling near $\varepsilon=0.07909$. It is clearly evident from the time series of the response oscillator in Fig.1(b) that the dynamics is basically a double scroll with intermittent jumps to the other double scroll in symmetrically opposite position. The time series of the driver in Fig.1(c) is double scroll chaotic which controls the response oscillator dynamics via unidirectional coupling.

(a)

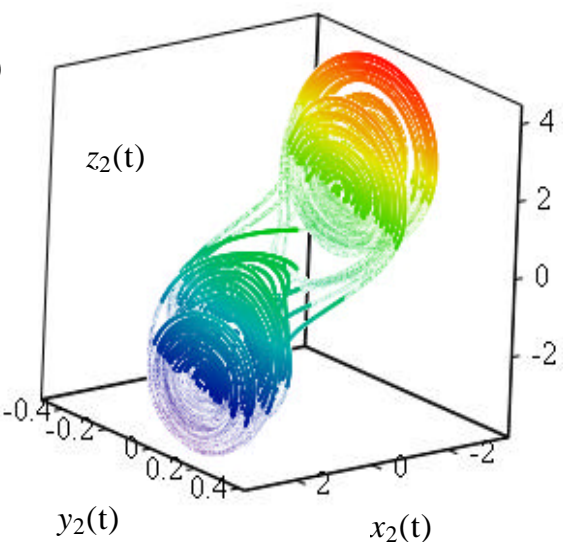

(b)

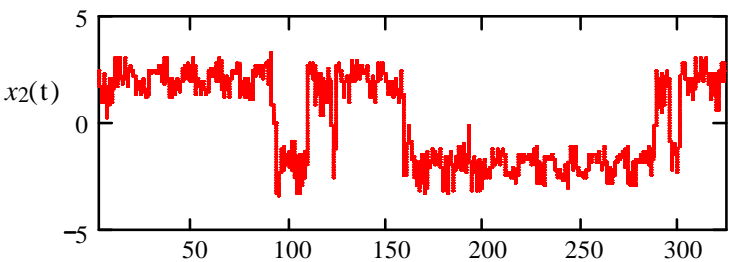

(c)

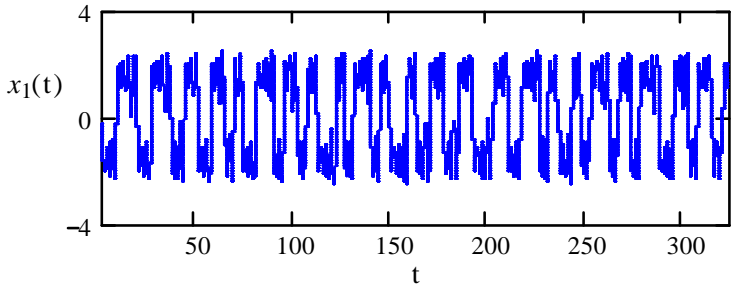

Fig.1. Multiscroll in coupled Chua oscillator: numerical results in (a) 3D 4scroll attractor, (b) $x_{2}(\mathrm{t})$ time series (red line) of 4-scroll attractor at the response, (c) $x_{1}(\mathrm{t})$ time series (blue line) of the driver. Parameters: driver oscillator, $\alpha_{1}=10.03138, \beta_{1}=10.77151, \gamma_{1}=0.33327, \mathrm{a}_{1}=-1.16971, \mathrm{~b}=-0.63055$ and response oscillator, $\alpha_{2}=9.39577, \beta_{2}=13.77255, \gamma_{2}=0.37518, a_{2}=-1.36903, b_{2}=-0.74268$, and coupling strength $\varepsilon=0.07909$.

In the single Chua oscillator, one could at most observe a double scroll (2-scroll) around the two symmetric saddle foci. As already mentioned, the trajectory of the double scroll is strongly repelled at the saddle focus origin, since its real eigenvalue $\gamma_{0}$ is always positive and $\left|\gamma_{0} / \sigma_{0}\right| \gg>1$. In a weakly coupled state, nine equilibrium points exist for the 6-dimensional coupled 
Chua oscillator. The saddle focus origin has six eigenvalues $\left(\gamma_{01}, \gamma_{02}, \sigma_{03} \pm j \omega_{03}, \sigma_{04} \pm j \omega_{04}\right)$. In addition, four pairs of equilibrium points exist as symmetric to the origin. Each of the symmetric pairs has six eigenvalues $\left(\gamma_{k 1}, \gamma_{k 2}, \sigma_{k 3} \pm j \omega_{k 3}, \sigma_{k 4} \pm j \omega_{k 4}\right)$ where the subscript $k(=1,2,3,4)$ denotes a symmetric pair of equilibrium points. Of them, two symmetric pairs each have a pair of negative real eigenvalues $\left(-\gamma_{k 1},-\gamma_{k 2}\right)$ and two pairs of complex eigenvalues $\left(\sigma_{k 3} \pm j \omega_{k 3}, \sigma_{k 4} \pm j \omega_{k 4}\right)$ with positive real parts $\left(\sigma_{k 3, k 4}\right)$. Four scrolls can exist at these two symmetric pairs of equilibrium points. The trajectory of a 4-scroll travels towards any of the two pairs of saddle foci along the eigendirection corresponding to the negative real eignenvalue and when it moves nearer to the saddle focus it spirals away in the 2D eigenplane corresponding to the complex conjugate eigenvalue with positive real part. The trajectory then moves to and spirals away from the other similar type of saddle focus (all negative real eigenvalues) in a similar fashion. For the remaining two symmetric pairs of equilibrium points, for any choice of parameters, at least one of the two real eigenvalues $\left(\gamma_{k 1}, \gamma_{k 2}\right)$ is always positive. Moreover, the real eigenvalues $\left(\gamma_{01}, \gamma_{02}\right)$ are always positive at the origin. At thes e five equilibrium points, the trajectory is strongly repelled due to the existence of at least one positive real eigenvalue and no scroll can be seen there. Thus we could at most observe 4scroll in coupled Chua oscillator. This is also confirmed by experiments presented in section 2.3 .

\subsection{Numerical Simulation: Coupled Modified Chua oscillator}

Here we introduce a new 3-scroll system in eq.2 which is derived by a small modification of the piecewise linear function of the original Chua model in eq.1, [cf. eq.1(g) and eq.2(d)]. Further the piecewise nonlinear function is now additive in eq.2(a). The modified Chua oscillator maintains three saddle focus type equilibrium points. However, in contrary to the original Chua oscillator, we find qualitatively similar type of eigenvalues for all three equilibrium points in the modified oscillator. The real eigenvalues are now negative for all three equilibrium points including the origin and the real parts of all complex conjugate eigenvalues are always positive maintaining the relation, $\left|\gamma_{\mathrm{j}} / \sigma_{\mathrm{j}}\right| \gg>1$ where $\mathrm{j}(=1,2,3)$ denotes a equilibrium point. Thus, in the modified Chua oscillator, the existence of 3-scroll dynamics becomes obvious: two scrolls around the symmetric saddle foci locations as usual and one additional scroll around the saddle focus origin as shown in the 3D plot in Fig.2(a). The governing equations of the 3 -scroll oscillator is given by

$$
\begin{aligned}
\frac{d x_{1}}{d t} & =\alpha_{1}\left[y_{1}-x_{1}+f\left(x_{1}\right)\right] \\
\frac{d y_{1}}{d t} & =x_{1}-y_{1}+z_{1} \\
\frac{d z_{1}}{d t} & =-\beta_{1} y_{1}-\gamma_{1} z_{1}
\end{aligned}
$$

(a)

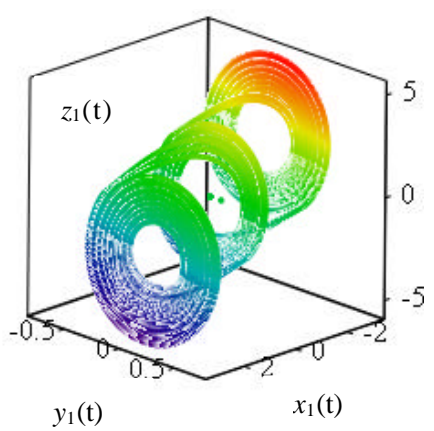

where $f\left(x_{1}\right)= \begin{cases}b_{1} x+\left(a_{1}-b_{1}\right) & \text { if } x_{1}<-1 \\ b_{1} x_{1} & \text { if }-1 \leq x_{1} \leq 1 \\ b_{1} x_{1}+\left(b_{1}-a_{1}\right) & \text { if } x_{1}>1\end{cases}$

(b)

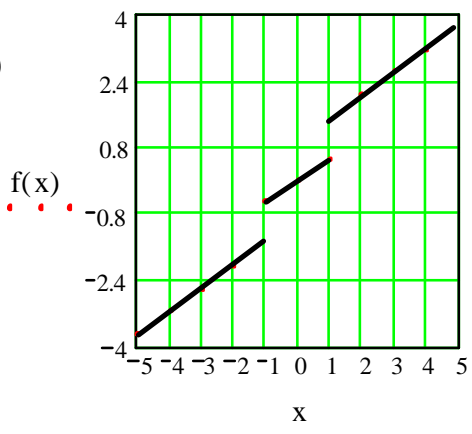

(c)

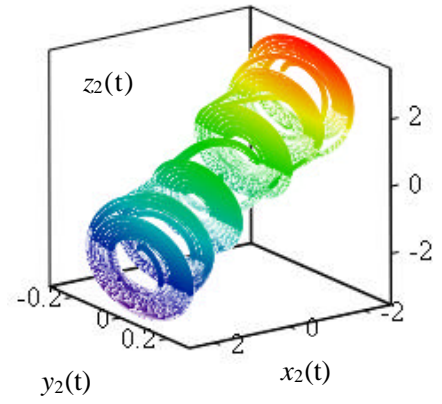

Fig.2. Modified Chua oscillator: (a) 3D 3-scroll attractor in single modified Chua oscillat or, parameters: $\alpha_{1}=7.9, \beta_{1}=15.67143, \gamma_{1}=0.005$, $a_{1}=-0.42857$, $\mathrm{b}_{1}=0.54457$, (b) modified piecewise linear function has three parallel linear parts with discontinuities at $\mathrm{x}= \pm 1$ (c) $3 \mathrm{D}$ 6-scroll attractor in coupled modified Chua oscillator. Parameters of response oscillator are identical to driver except $\alpha_{2}=1.2$. Coupling strength $\varepsilon=0.2$ for 6 -scroll. 
The piecewise linear function of the modified oscillator, defined in eq.(2d), is plotted in Fig.2(b) which shows three linear parts with two discontinuities at $x= \pm 1$. In mark departure from the original Chua oscillator, the trajectory in Fig.2(a) now rotates around all three saddle foci including the saddle focus origin as explained above. The proposed unidirectional coupling scheme via the x-coordinate is then applied to the modified Chua oscillator and we are able to generate a 6-scroll as shown in Fig.2(c) in the intermittency regime of weaker coupling. Again, the 3-scroll attractor occasionally jumps to another 3-scroll attractor in a symmetric location in 6-dimension. Hence, we conclude that any $n$-scroll $(n=2,3, \ldots)$ attractor in a reflection symmetric system like the Chua oscillator when forced into a similar oscillator in a resting state, leads to generate a $2 n$-scroll in an intermittency regime of weaker coupling. In the next section, an experiment on 4-scroll dynamics is described to support our coupling scheme for the generation of $2 \mathrm{n}$-scroll.

\subsection{Experiment: 4-scroll in coupled Chua circuit}

A coupled Chua circuit is shown in Fig.3 where each oscillator OS-1 (OS-2) is designed by using resistance $\mathrm{R}_{1}\left(\mathrm{R}_{8}\right)$, capacitors $\mathrm{C}_{1}\left(\mathrm{C}_{3}\right)$ and $\mathrm{C}_{2}\left(\mathrm{C}_{4}\right)$, and inductance $\mathrm{L}_{1}\left(\mathrm{~L}_{2}\right)$ and two op-amps U1-U2 (U3-U4). The $\mathrm{r}_{01}$ and $\mathrm{r}_{02}$ are the inductor leakage resistances. The op-amps U1-U2 (U3-U4) simulate the piecewise linear function defined in eq.1(g). The unity gain opamp U5 sets the unidirectional coupling between OS-1 and OS-2 while the resistance $\mathrm{R}_{\mathrm{C}}$ decides the coupling strength. All component values are noted in the circuit. The oscillator OS-1 is set for double scroll chaos and forced into the oscillator OS-2, which is in a resting state when uncoupled. A state of synchrony can be reached for a strong coupling when both amplitude and phase of the driver and the response are almost identical. As the coupling strength is decreased, transition to phase synchrony is observed Roy, Chakraborty and Dana, 2003] via successive lag synchronization and an intermittency regime. In this intermittency regime, the 4-scroll attractor is observed where the trajectory switches intermittently between two double scroll as shown in Fig.4(a). The voltages $V_{\mathrm{C} 1, \mathrm{C} 3}(\mathrm{t}), V_{\mathrm{C} 2, \mathrm{C} 4}(\mathrm{t})$ at the nodes of $\mathrm{C}_{1,3}, \mathrm{C}_{2,4}$ are monitored using a 2-channel digital oscilloscope (Tektronix, TDS 220,100MHz) with 2.5k data record length and maximum sampling speed $1 \mathrm{GS} / \mathrm{s}$.

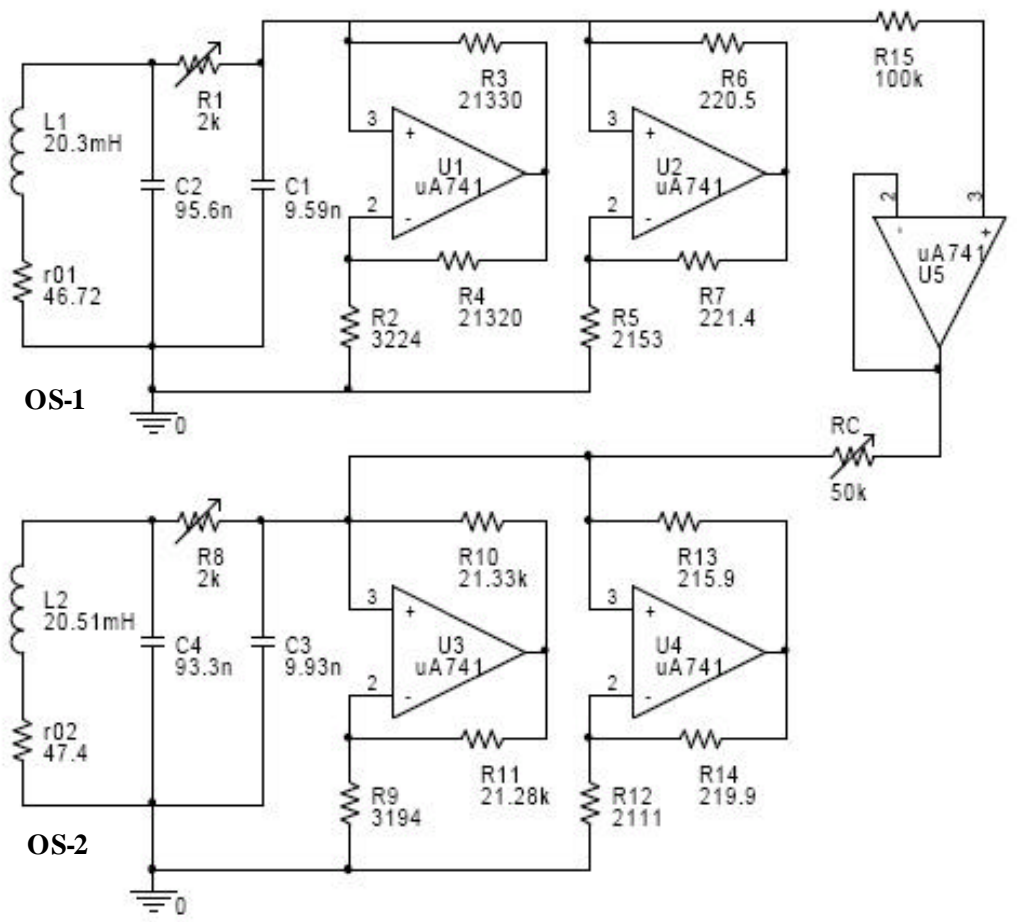

Fig.3. Two unidrectionally coupled Chua oscillators: power supply $\pm 9 \mathrm{~V}$. OS-1 is the driver and OS-2 is the response oscillator. All component values are noted in the diagram as measured using a standard LCR-Q bridge (APLAB 4910, 1kHz).

All circuit components are kept fixed except $R_{1}, R_{8}$ and $R_{C}$. The resistances $R_{1}$ and $R_{8}$ decide the driver and response dynamics respectively. The resistance $R_{C}$ is varied to set the coupling strength. In the uncoupled state, the driver is double scroll chaotic for $R_{1}=1482 \Omega$, and the response is in a resting state or at a stable focus for $R_{8}=1636 \Omega$. A state of synchrony between the driver and the response is established for strong coupling when the coupling resistance is as small as $R_{C} \leq 150 \Omega$. Once the coupling strength is decreased by increasing the coupling resistance $R_{C}$, the 4-scroll chaos appears in the response oscillator for an intermediate range of coupling strength near $\mathrm{R}_{\mathrm{C}}=16.59 \mathrm{k} \Omega$. The $3 \mathrm{D}$ trajectory of the 4-scroll and the time series of $V_{\mathrm{C} 3}(\mathrm{t})$ are shown in Fig.4. The 3D trajectory is plotted using measured voltages $V_{\mathrm{C} 3}(\mathrm{t}), V_{\mathrm{C} 4}(\mathrm{t})$ and delayed voltage $V_{\mathrm{C} 3}(\mathrm{t}-2)$ along the $\mathrm{X}$ axis, the Y-axis and the Z-axis respectively. By comparison of Fig.1 and Fig.4, one can easily find a very good agreement between the numerical results and the experiment. 

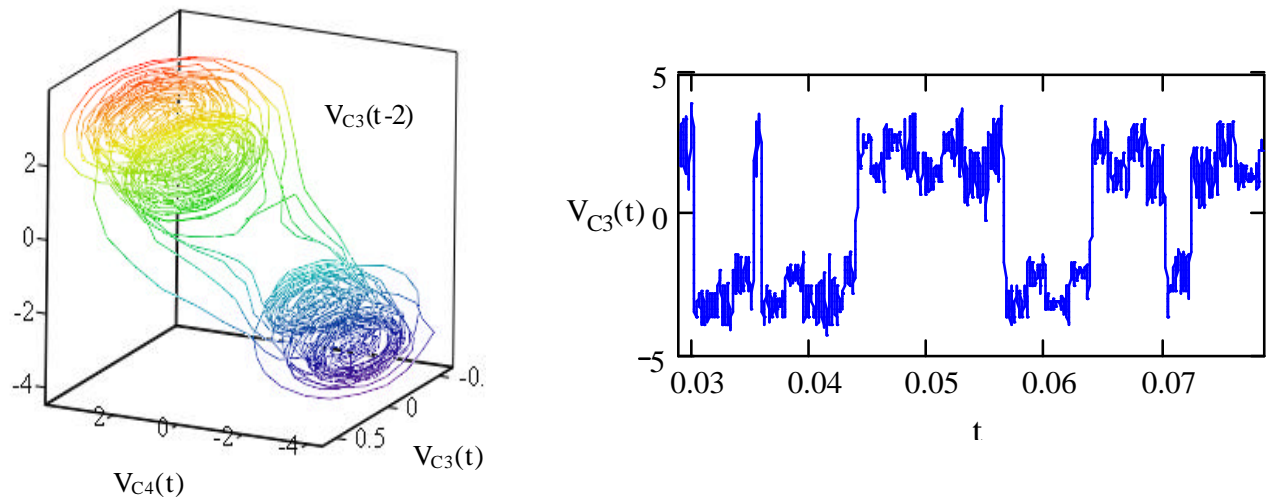

Fig.4. Experimental 4-scroll in coupled Chua circuit: $R_{1}=1482 \Omega$ and $R_{8}=1636 \Omega$ and $R_{C}=16.59 k \Omega$. (a) $3 D$ trajectory of 4scroll plotted using $\mathrm{V}_{\mathrm{C} 3}(\mathrm{t}), \mathrm{V}_{\mathrm{C} 4}(\mathrm{t})$ and time delayed $\mathrm{V}_{\mathrm{C} 3}(\mathrm{t}-2)$, (b) experimental time series in blue of $\mathrm{V}_{\mathrm{C} 3}(\mathrm{t})$ showing 4-scroll dynamics.
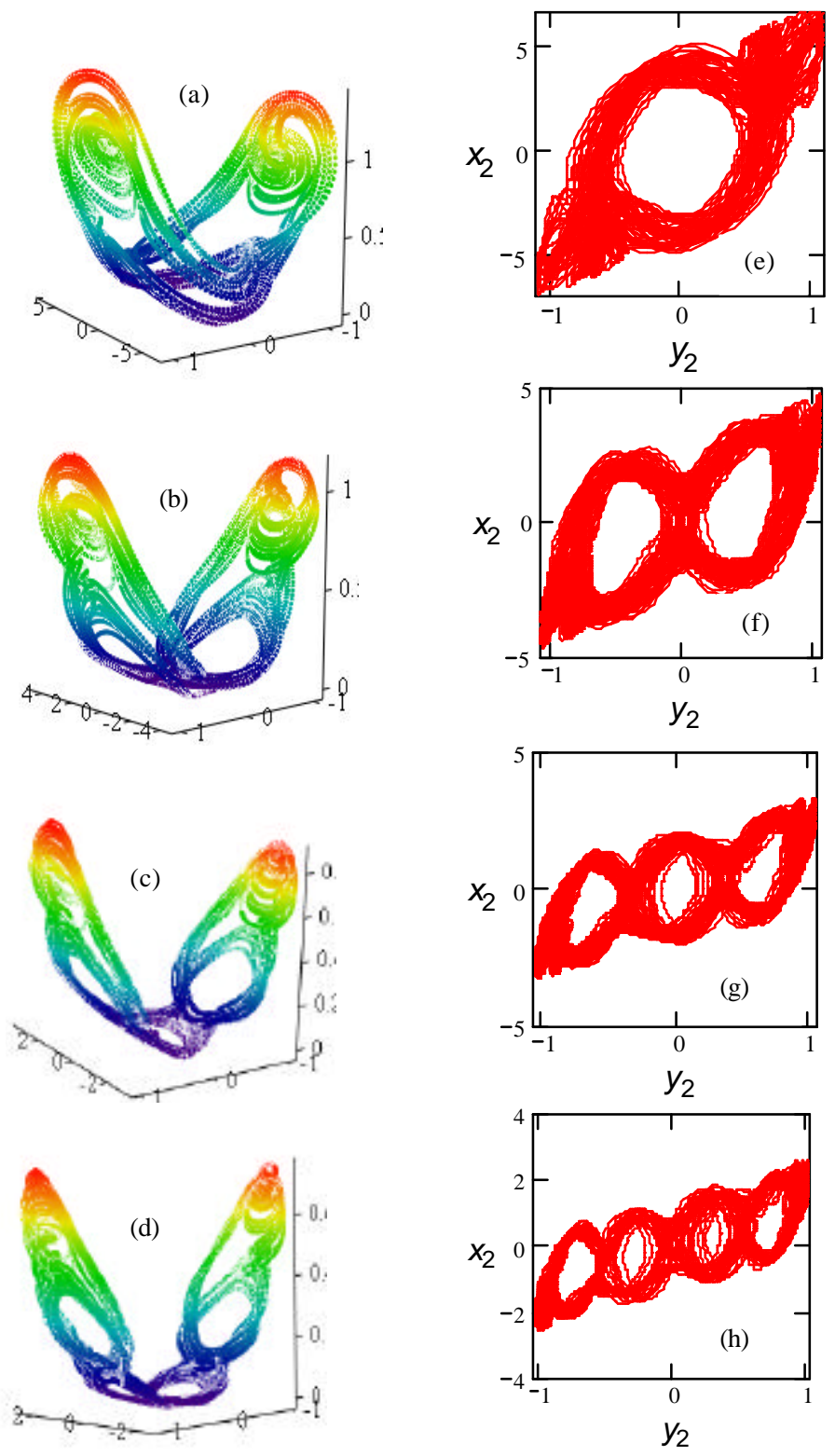

Fig.5. Multiscroll in coupled Lorenz system: numerical results, $b=8 / 3, \sigma=10, \mathrm{r}=35, \mathrm{r}_{2}=1.15$; the $3 \mathrm{D}$ response attractors in (a)-(d), corresponding 2D phase portraits in column (e)-(h). The 3-scroll in (a) \& (e) for $\varepsilon=2.85$, 4-scroll in (b) \& (f) for $\varepsilon=1.9,5$-scroll in (c) \& (g) for $\varepsilon=1.313$ and 6 -scroll in (d) \& (h) for $\varepsilon=0.95$. The $3 \mathrm{D}$ attractors are plotted using $\mathrm{x}_{2^{-}}, \mathrm{y}_{2}$ - and $\mathrm{z}_{2}$-variables of the response oscillator. 


\section{Two Unidirectionally Coupled Lorenz Systems}

The proposed unidirectional coupling scheme is extended to the Lorenz system. The driver Lorenz system is set for the usual butterfly-type attractor while the response system remains in a resting state when uncoupled. Interestingly, we find multiscroll attractors at the response system in the weaker coupling regime. In fact, multiscroll attractors (3-, 4, 5-, 6-scroll) evolve as one after another additional scroll emerges with a gradual decrease in the coupling strength as shown in Fig.5. It appears as if a hidden multiscroll structure unfolds in the response oscillator, which is otherwise dormant in uncoupled state, when a butterfly type Lorenz attractor is forced into it and the forcing strength is weakened.

\subsection{Numerical Simulation}

The governing equations of two coupled Lorenz systems in drive-response mode are given by

$$
\begin{aligned}
& \frac{d x_{1}}{d t}=\sigma\left(y_{1}-x_{1}\right) \\
& \frac{d y_{1}}{d t}=r_{1} x_{1}-y_{1}-x_{1} z_{1} \\
& \frac{d z_{1}}{d t}=-b z_{1}+x_{1} y_{1} \\
& \frac{d x_{2}}{d t}=\sigma\left(y_{2}-x_{2}\right)+\varepsilon\left(x_{1}-x_{2}\right) \\
& \frac{d y_{2}}{d t}=r_{2} x_{2}-y_{2}-x_{2} z_{2} \\
& \frac{d z_{2}}{d t}=-b z_{2}+x_{2} y_{2}
\end{aligned}
$$

Equations 3(a)-(c) represent the driver and equations 3(d)-(f) represent the response oscillator. The parameters are chosen as $\sigma=10$ and $\mathrm{b}=8 / 3$ for both the oscillators except $r_{1}$ and $r_{2}$. The parameter $r_{1}=35$ is chosen to set the driver dynamics to the butterfly-type attractor, while $r_{2}=1.15$ is chosen to set the response oscillator in a resting state when uncoupled. The choice of $r_{1}$ and $r_{2}$ is arbitrary; they are so chosen that the driver is double scroll chaotic and the response is in a resting state. $\varepsilon$ defines the coupling strength. For weaker coupling $\varepsilon=2.85$, we observed $n=3$-scroll as shown in Fig.5(a) \& 5(e). By weaker coupling we mean that it is much lower than what is needed for complete synchronization. In fact, the coupling strength is very large $(\varepsilon>100)$ for complete synchronization in our proposed coupling scheme. The phase portrait in Fig.5(e) shows a 3-scroll: one large scroll is clearly seen around the saddle origin and two smaller scrolls at symmetric locations but they are not very clear from the 2D projection. However, the smaller symmetric scrolls are clearly seen in the 3D plot of Fig.5(a). Then, by gradually decreasing the coupling strength, one can see the emergence of one after another additional scroll, as shown in the 3D plots of Fig.5(a)-(d) and in their respective phase portraits in Fig.5(e)-(h). The outermost smaller scrolls are always present although their size decreases with decreasing coupling strength; only inner scrolls are added to the attractor one after another until we find a full bloom of the hidden structure as a 6-scroll. The size of the inner scrolls decreases too with its increasing number. It is worth mentioning that such multiscroll can be seen for the x-coordinate coupling only, coupling via any other coordinate like $y$ - or zcoordinate fails to show such a multiscroll. In the next section, we explain the evolution of the multiscroll using eigenvalue analysis and schematic diagrams of multiscroll trajectories.

\subsection{Origin of Multiscroll in the Coupled Lorenz System}

In a single Lorenz system, there are three equilibrium points: two saddle foci symmetric to a saddle origin. The trajectory of a typical butterfly attractor in the Lorenz system rotates around either of the symmetric saddle foci. In two coupled Lorenz systems, the response system develops a state of complete synchrony with the driver for a strong coupling above a threshold. Accordingly, the coupled 6-dimensional system also has three equilibrium points (two saddle foci and one saddle origin) for very strong coupling, while nine equilibrium points (three pairs of symmetric saddle foci and three saddle including the saddle origin) exist for very weak coupling. We calculated the eigenvalues of all the equilibrium points with varying coupling coefficients and thereby identified the type of the equilibrium points either as saddle or saddle focus. We find that the coupled system continues with three equilibrium points with decreasing coupling strength until a critical coupling, $\varepsilon=\varepsilon_{C 1}=\sigma\left(r_{2}-1\right)$ is reached, when a bifurcation is seen. For the selected parameters $\left(\sigma=10, b=8 / 3, \mathrm{r}_{1}=35\right.$ and $\left.\mathrm{r}_{2}=1.15\right)$ of the coupled Lorenz system, this bifurcation point is reached at a critical coupling $\varepsilon_{\mathrm{Cl}}=1.5$, which is shown in Fig.6(a). In Figs.6(a) and 6(b), $\mathrm{y}_{2}^{\mathrm{e}}$ is plotted in dependence on the coupling strength $\varepsilon$, where $\mathrm{y}_{2}=\mathrm{y}_{2}{ }_{2}^{\mathrm{e}}$ represents the state variable $\mathrm{y}_{2}$ of the response system at the equilibrium point and superscript $e$ denotes an equilibrium point. Three equilibrium lines (two symmetric lines with open, solid squares and a horizontal solid line) are seen in Fig.6(a) for $\varepsilon>1.5$. The outermost two lines (with open and solid squares) at symmetrically opposite positions are of saddle focus type for all $\varepsilon$ as indicated by a rotating arrow and denoted by the letter $s f$. The horizontal zero line is the saddle origin line denoted by the letter $s$. Two additional saddle lines emerge (symmetric dotted lines with open and solid circles) at the bifurcation point of the first critical coupling $\varepsilon_{C 1}=1.5$, which continues to remain of saddle type for all $\varepsilon<1.5$. Thus the coupled system has five equilibrium points for $\varepsilon<1.5$ : two symmetric saddle foci as usual and two symmetric saddle points in addition to the saddle origin until reached a second critical coupling $\varepsilon=\varepsilon_{\mathrm{C} 2}$. At the second critical coupling, $\varepsilon=\varepsilon_{C_{2}}$, two additional pairs of symmetric equilibrium lines (a symmetric pair of solid lines with open, closed diamond and another symmetric pair of dotted lines with open, closed triangle) emerge as shown in Fig.6(b), which is an 
enlarged version of Fig.6(a) in the coupling range, $0.01<\varepsilon<0.05$. To calculate the second critical coupling, we define a quantity $\Delta$ in terms of the coupled Lorenz system parameters and coupling strength as given by

$$
\begin{aligned}
& \Delta=\frac{P^{3}}{27}-\frac{Q^{2}}{4} \\
& \text { where } P=B-\frac{A^{2}}{3} ; Q=C-\frac{A B}{3}+\frac{2 A^{3}}{27} ; A=\frac{\varepsilon}{\sigma+\varepsilon} ; B=\frac{\sigma b\left(\mathrm{r}_{1}-\sigma^{\prime}\right)}{\sigma+\varepsilon} ; C=\frac{\varepsilon b b^{\prime}}{\sigma+\varepsilon} ; \sigma^{\prime}=\frac{\sigma+\varepsilon}{\sigma} ; b^{\prime}= \pm \sqrt{b\left(r_{1}-1\right)}
\end{aligned}
$$

The second critical coupling or the second bifurcation point is obtained where $\Delta$ changes its sign. For the chosen coupled system parameters given above, the second critical coupling is found as $\varepsilon_{\mathrm{C} 2}=0.0352743$ as shown in Fig.6(b). The two new symmetric pairs of equilibrium points for $\varepsilon<\varepsilon_{\mathrm{C} 2}$ are all saddle focus type, when the coupled Lorenz system has finally nine equilibrium points: three symmetric pairs of saddle foci and three saddle including the saddle origin. However, the coupling below $\varepsilon=0.87$ is not interesting from the viewpoint of multiscroll dynamics, since no additional scroll is seen below this. For the whole range of coupling, the two outermost symmetric lines with open and solid squares are the saddle foci lines, and the two symmetric dotted lines with open and solid circles are the saddle lines as shown in both Fig.6(a) and Fig.6(b).

In this background of evolving equilib rium points and their stability with coupling, we explain the origin of the multiscroll dynamics using schematic diagrams of multiscroll trajectories in Fig.7. Each circle is a schematic line drawing of each scroll or a bundle of trajectories around an equilibrium point. The size of the circles is arbitrary and never representing the true size of a scroll. Notations $s$ and $s f$ denote saddle and saddle focus respectively as mentioned above. A dotted horizontal line is drawn to mark the first critical coupling $\left(\varepsilon_{\mathrm{Cl}}=1.5\right)$ which is involved with the birth or death of two additional saddle points. For strong coupling $\varepsilon>100$, the response oscillator is in synchrony with the driver. The trajectory makes a 2 -scroll around two symmetric saddle foci and it crosses from one scroll to the other near the saddle origin as shown in Fig.7(a) and it appears as the two winged typical butterfly attractor of Lorenz system. In a range of weaker coupling strength, $5.0>\varepsilon>1.5$, the response oscillator shows n=3-, 4-scroll dynamics. Figures 5(a) \& 5(e) shows a 3-scroll for $\varepsilon=2.85$ : one big scroll around the saddle origin and two smaller scrolls each around each of the symmetric saddle foci. The schematic of 3-scroll is shown in Fig.7(b) where $s$ and $s f$ are placed near the middle of each circle to indicate that the scrolls encircle their respective equilibrium points. By further lowering the coupling strength, one more scroll appeared: the trajectory of the 4-scroll now makes two inner big scrolls while switching between the outer smaller scrolls around two saddle foci as seen in Figs.5(b) \& 5(f). Two big inner scrolls now exist in Fig.7(c) that cross each other near the saddle origin marked by an arrow. Below the first critical line $\left(\varepsilon_{\mathrm{Cl}}=1.5\right)$, two more saddle points symmetric to the saddle origin emerge. Accordingly, we could find a 5-scroll for $\varepsilon=1.313$ and a 6-scroll for $\varepsilon=0.95$ as shown in Fig.5(g) and 5(h) respectively. The schematic diagrams of a 5-scroll and a 6-scroll are shown in Fig.7(d) and 7(e) respectively. Two smaller outer scrolls around the saddle foci and three big inner scrolls around the three saddle points are seen in the 5-scroll attractor in Fig.7(d). Two smaller outer scrolls as usual around symmetric saddle foci and four big inner scrolls are seen for a 6-scroll in Fig.7(e). Instead of three inner scrolls encircling three saddles as seen in Fig.7(d), the trajectory of four inner scrolls now crosses each other near the saddle points. The trajectory of the multiscroll actually moves towards the saddle points before being repelled by themselves whose positions are indicated by arrows in the schematic diagram in Fig.7(c) and $7(\mathrm{e})$.
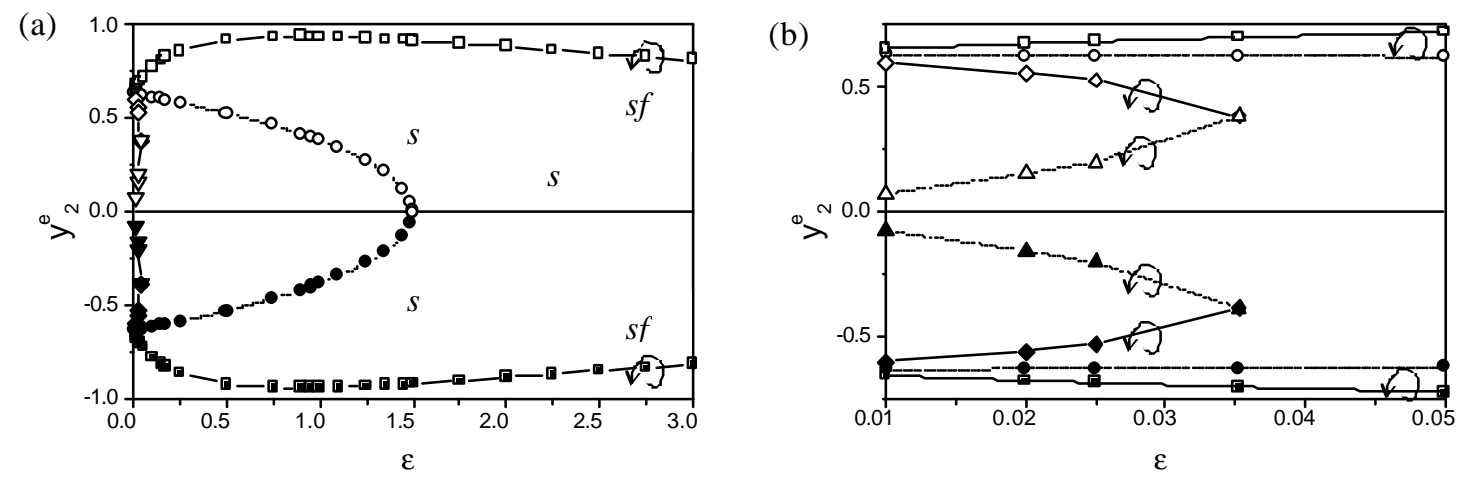

Fig. 6. Evolution of equilibrium points: $b=8 / 3, \sigma=10, \mathrm{r}_{1}=35, \mathrm{r}_{2}=1.15$. The $\mathrm{y}_{2}^{\mathrm{e}}$ is plotted with coupling, (a) for the coupling range, $\varepsilon=0-3.0$, which is enlarged in (b) for the coupling range $\varepsilon=0.01-0.05$. Rotating arrows indicate saddle foci as also denoted by $s f$ while a saddle is denoted by $s$.

It may be noted here that we obtain two bifurcation points at two different coupling strengths where new equilibrium points emerge. We have identified the nature of all the equilibrium points using eigenvalue analysis. In the first bifurcation point or the first critical coupling, two new saddle points emerge in addition to the saddle origin. Beyond this bifurcation point, one after another additional scroll appears with decreasing coupling until we observe at most 6-scrolls: four inner scrolls and two outermost scrolls as described in Fig.7. The outermost scrolls around the outer two saddle foci are always present for any coupling strength while the four inner scrolls appears at different coupling strength when it is gradually decreased. However, above the first critical coupling, the number of inner scrolls is only restricted to two scrolls which cross near the saddle origin 
as seen in Fig.7(c). Two more inner scrolls are only seen below the first critical coupling when two additional saddle points emerge. It may be noticed that the four inner scrolls either revolve around a saddle or made a crossing near a saddle where the trajectory is actually repelled by the saddle. We observe a second bifurcation point for a very low coupling as shown in Fig.6(b) at $\varepsilon \leq \varepsilon_{\mathrm{C} 2}=0.0352743$ for the specific choice of system parameters as calculated by using eq.4. But this bifurcation point is not interesting from the viewpoint of generation of any new scroll. From this eigenvalue analysis of all equilibrium points for coupling strength ranging from very strong to very weak coupling, we conclude that the emergence of the inner scrolls is a process, since no new bifurcation is seen during their evolutions except the first critical coupling. The first critical coupling originates additional saddle points which allow the emergence of larger number of scrolls than just two.

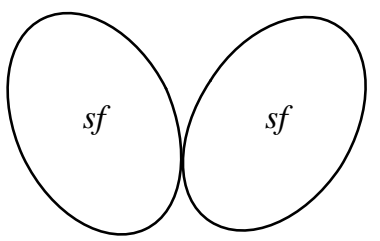

(a) $\varepsilon=100$

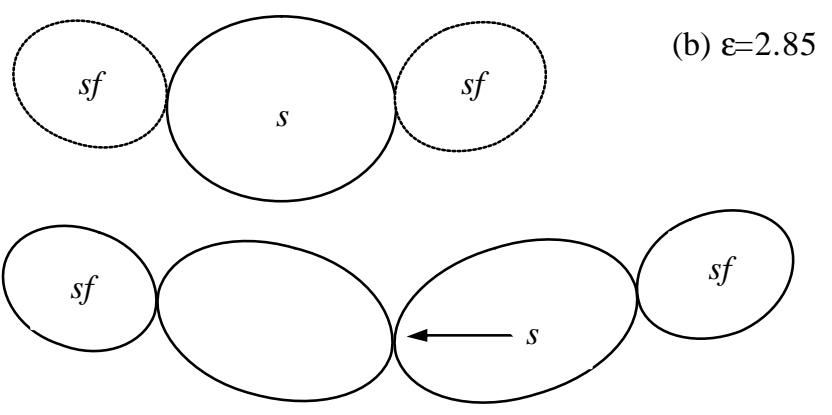

(c) $\varepsilon=1.9$

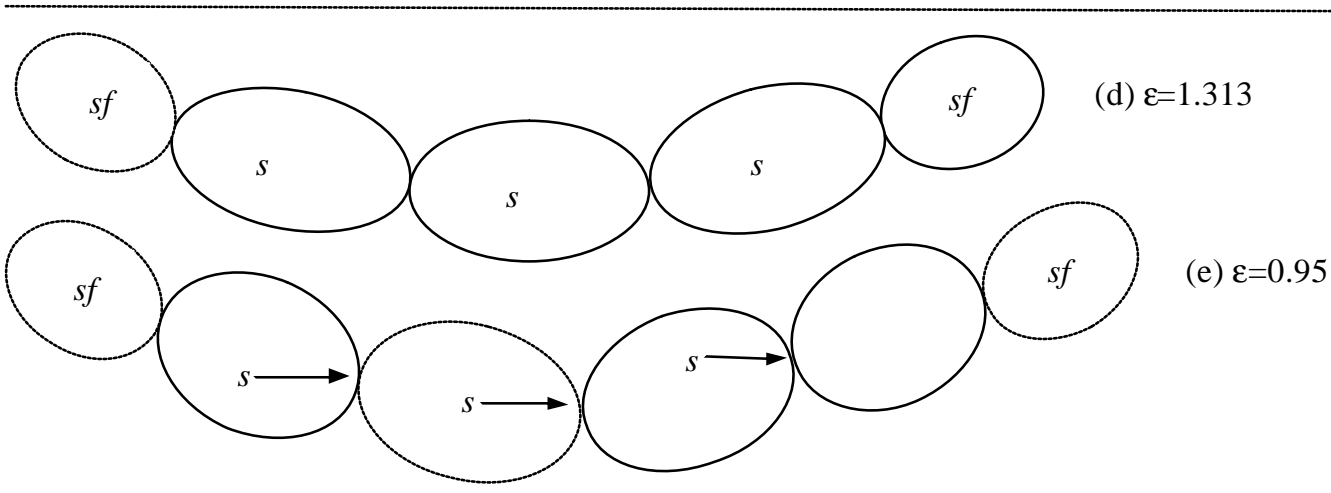

Fig.7. Schematics of multiscrolls: $b=8 / 3, \sigma=10, \mathrm{r}_{1}=35, \mathrm{r}_{2}=1.15$, (a) $\varepsilon=100, \mathrm{n}=2$-scroll, (b) $\varepsilon=2.85, \mathrm{n}=3$-scroll, (c) $\varepsilon=1.9, \mathrm{n}=4$-scroll, (d) $\varepsilon=1.313$, $\mathrm{n}=5$ scroll, (e) $\varepsilon=0.95, \mathrm{n}=6$-scroll. Notations $s$ and $s f$ indicate saddle and saddle focus. In reality, trajectories never intersect as appeared in the diagram during change over from one scroll to the other. The trajectories also never touch the saddle points. These are highly simplified schematics.

A numerical bifurcation diagram is presented in Fig.8 to describe the evolution of the scrolls with decreasing coupling strength. The local maxima of the $y_{2}$-variable of the response Lorenz system represented by eqn.(3) is plotted with coupling strength. We tried to capture the open space inside each scroll as they emerge with coupling strength as shown in Fig.5. The open space inside a scroll is represented by white dots while the trajectories are indicated by black dots. We chose an appropriate Poincáre plane which is able to identify only the inner scrolls. Two outer symmetric scrolls around the saddle foci cannot be captured in the same plane, since we find it difficult to assign a unique Poincáre plane so as to capture both the big inner scrolls and the outermost two scrolls. However, it may be noticed in Fig. 5 that the outer two scrolls could be easily identified from the 3D projection, which are always present for any coupling strength and they never bifurcate. Hence we remain concerned only with the emergence of the inner scrolls or the unfolding of one after another additional scroll with gradual decrease in coupling. This phenomenon is clearly revealed in the bifurcation diagram. Figure 8(a) shows the bifurcation diagram for the coupling strength in the range of 0.25 to 5.0. It clearly shows one open space for the coupling range $5.0>\varepsilon>2.68$ as indicated by a triangular white space denoted by region 1 . The triangular open space, region 1 , terminates near $\varepsilon=2.68$ as indicated by an arrow where the trajectories crosses each other. Accordingly, a 3-scroll with a big inner scroll is seen for $\varepsilon=2.85$ in region 1. Of course, the two outermost scrolls always exist around the symmetric saddle foci, although we do not observe them in the bifurcation diagram. Two new open spaces marked by region 2 and region 3 started appearing near $\varepsilon=2.63$ and this indicates the evolution of two inner scrolls and they continue until $\varepsilon=1.67$ below which three open spaces started to 
evolve, however, they are not very clearly visible in Fig.8(a). Three open spaces are clearly seen in Fig.8(b) for $1.17<\varepsilon<1.38$, which indicates the evolution of three scrolls. Figure 8(a) is enlarged in Fig.8(b) for the coupling range, $0.75<\varepsilon<2.0$. For further low coupling in the range of $0.96>\varepsilon>0.8$, four open spaces are seen, which clearly indicates the existence of four inner scrolls. It may be noted that the open spaces evolve one after another with an intermediate transition region of coupling where crossing of trajectories are seen with black dots. No clear scroll is actually seen in these transition regimes. The trajectories reorganize themselves in the transition regions before reappearing with an additional scroll in the open regions of coupling. For $\varepsilon<0.87$, the whole space is blurred with black dots and hence no clear scroll is seen any more. The ranges of coupling strength as indicated in the bifurcation diagrams are found consistent with the evolution of multiscrolls in Fig.5.

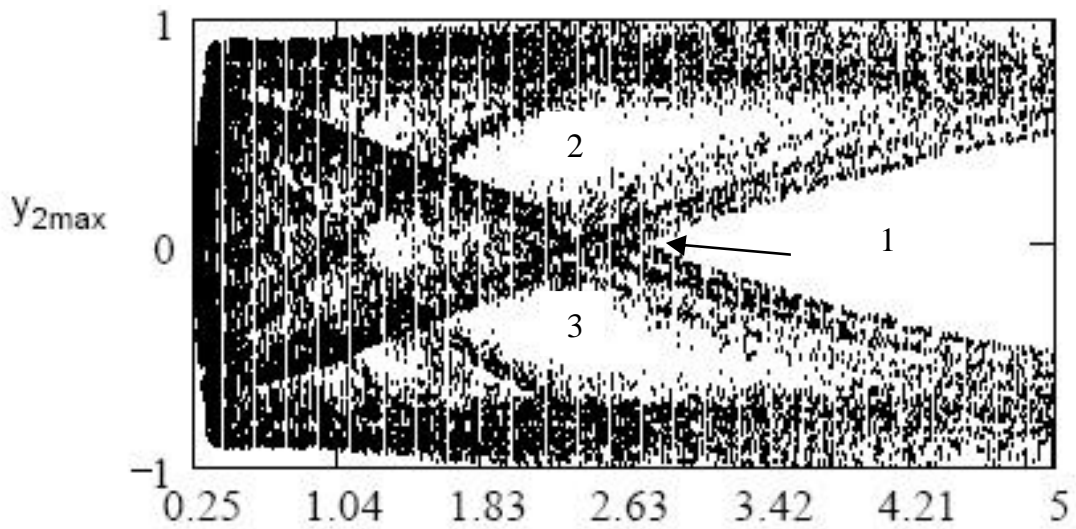

(a)

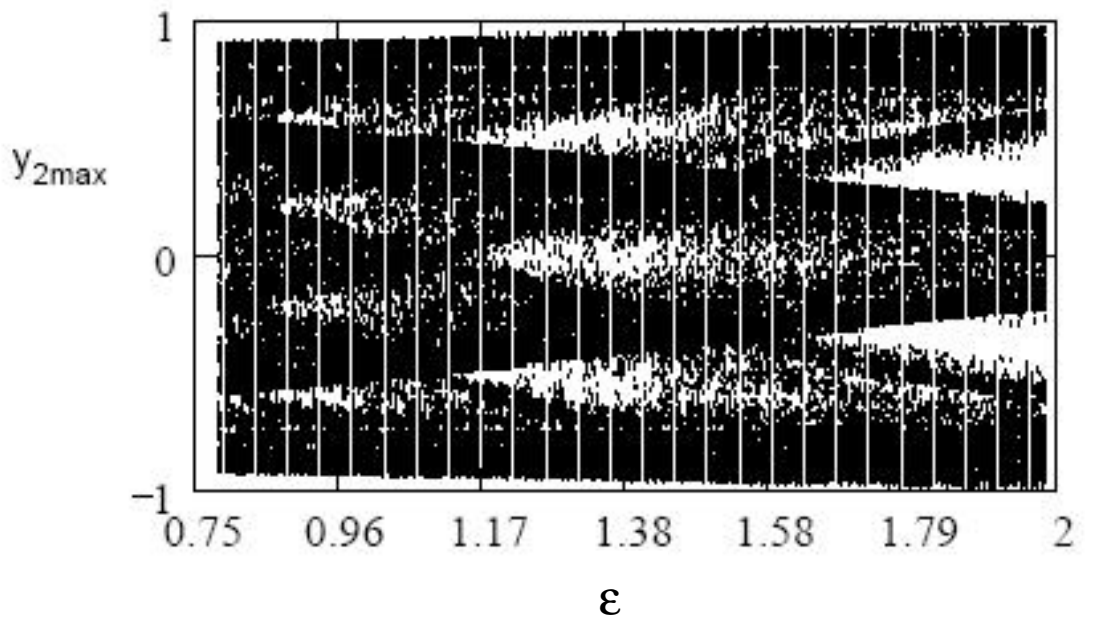

(b)

Fig.8. Bifurcation diagram: $b=8 / 3, \sigma=10, \mathrm{r}_{1}=35, \mathrm{r}_{2}=1.15 ; \mathrm{y}_{2 \max } \mid$ is plotted against coupling strength $(\varepsilon)$. (b) is the enlarged version of (a) in the coupling limit $0.75<\varepsilon \leq 2.0$. Emphasis is given on the evolution of the inner scrolls, Poincáre plane is so chosen that the inner scrolls are clearly identified. Outermost two scrolls are not observable in the same Poincáre plane.

\subsection{Experimental evidence of multiscroll in coupled Lorenz circuits}

An electronic analog of two coupled Lorenz systems defined by eq.(3) is designed for finding experimental evidences of multiscroll dynamics. We scale down the state variables in eq.(3) to keep the size of the butterfly attractor within the limit of the power supply voltage level. Otherwise the circuit goes to the saturation limit of the power supply and stops oscillating. A new set of state variables is thus defined by $u_{1,2}=x_{1,2} / 10, v_{1,2}=y_{1,2} / 10$ and $w_{1,2}=z_{1,2} / 10$. The electronic analog of the coupled Lorenz system is shown in Fig.9. The driver (response) Lorenz circuit is designed using three op-amps U1-U3: $\mu$ A741 (U6$\mathrm{U} 8: \mu \mathrm{A} 741$ ) and two analog multipliers U4-U5:AD633 (U9-U10:AD633) with associated resistances $\mathrm{R}_{4}-\mathrm{R}_{7}\left(\mathrm{R}_{8}-\mathrm{R}_{14}\right)$ and capacitors $\mathrm{C}_{1}-\mathrm{C}_{3}\left(\mathrm{C}_{4}-\mathrm{C}_{6}\right)$. The driver is unidirectionally coupled to the response oscillator by the op-amp U11: $\mu \mathrm{A} 741$ and the resistance $R_{17}$. The resistance $R_{17}$ decides the coupling strength of the driver-response system. 


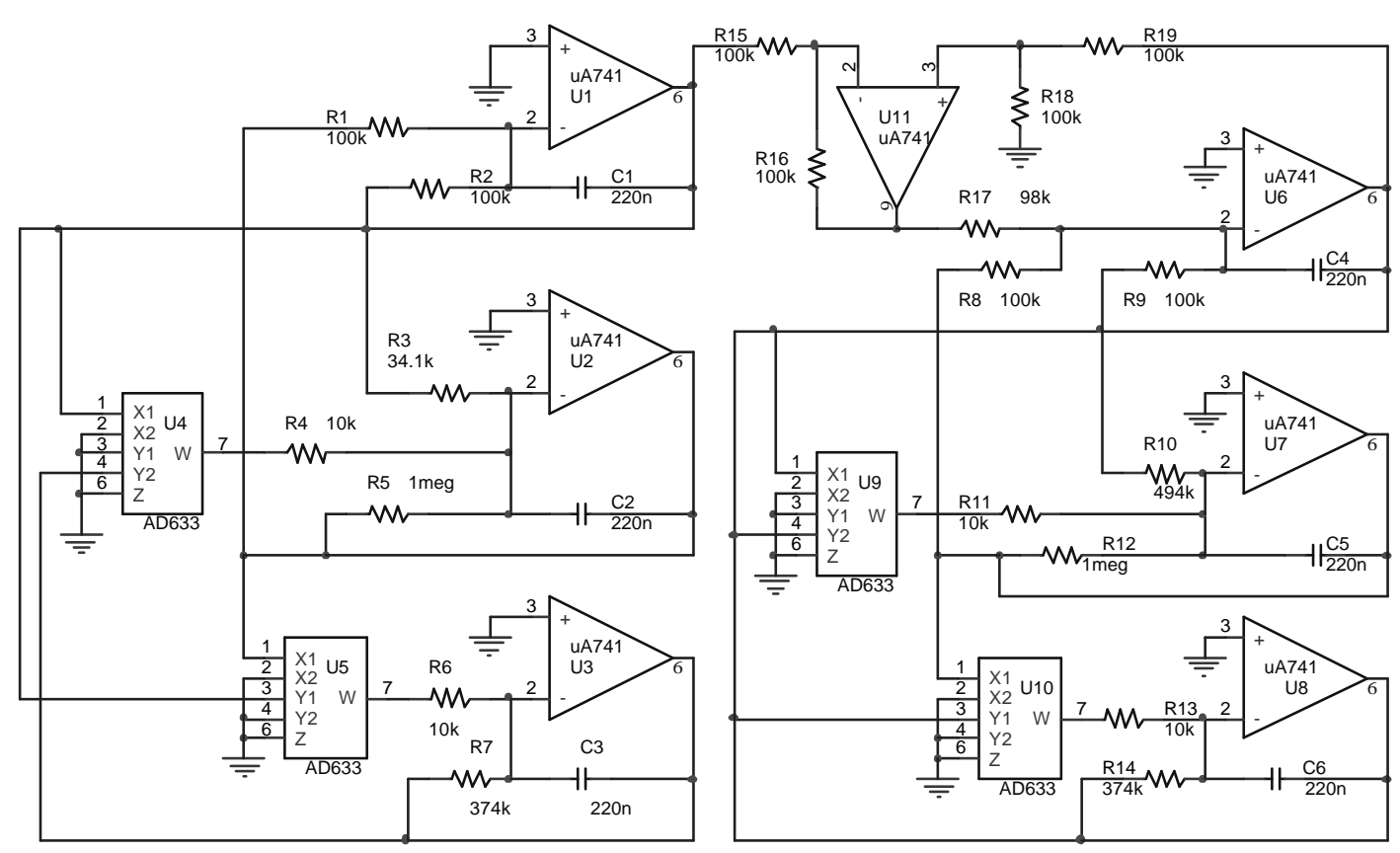

Fig.9. Two coupled Lorenz oscillators: Power supply is $\pm 15 \mathrm{~V}$. The values of selected component are noted against each component . Unit of resistancein ohm, capacitor in farad.
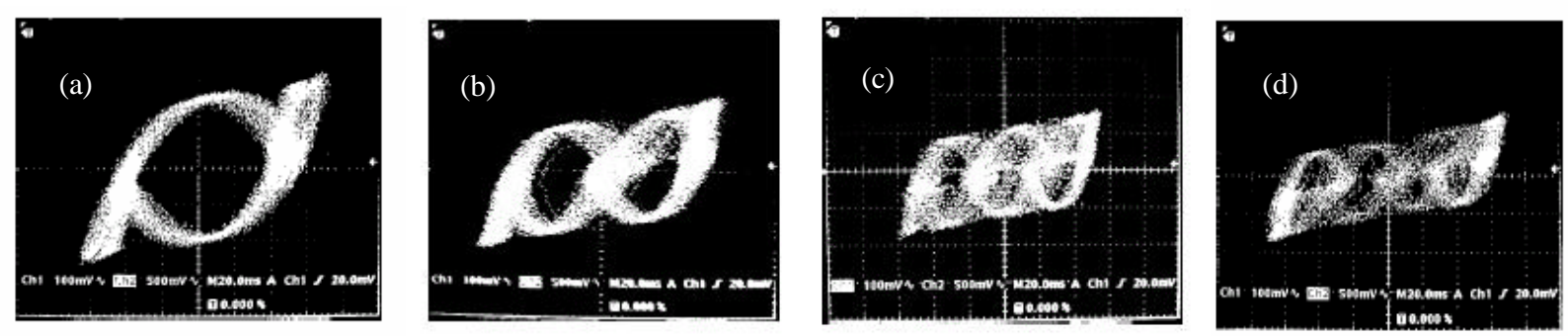

Fig.10. Experimental multiscroll attractor in response Lorenz system: 2-D phase portraits of multiscroll in (a)-(d): (a) $R_{7}=98 \mathrm{k} \Omega, n=3$-scroll, (b) $\mathrm{R}_{17}=264.2 \mathrm{k} \Omega, \mathrm{n}=4$-scroll, (c) $\mathrm{R}_{17}=375.5 \mathrm{k} \Omega$, $\mathrm{n}=5$-scroll, (d) $\mathrm{R}_{17}=437.8 \mathrm{k} \Omega$, $\mathrm{n}=6$-scroll.

Using Kirchoff's current and voltage laws, the governing equations of the coupled Lorenz circuit are defined by

$$
\begin{aligned}
\frac{d u_{1}}{d t} & =\tau\left[\sigma\left(v_{1}-u_{1}\right)\right] \\
\frac{d v_{1}}{d t} & =\tau\left[r_{1} u_{1}-v_{1}-u_{1} w_{1}\right] \\
\frac{d w_{1}}{d t} & =\tau\left[-b w_{1}+u_{1} v_{1}\right]
\end{aligned}
$$

$$
\begin{aligned}
& \frac{d u_{2}}{d t}=\tau\left[\sigma\left(v_{2}-u_{2}\right)+\varepsilon\left(u_{1}-u_{2}\right)\right] \\
& \frac{d v_{2}}{d t}=\tau\left[r_{2} u_{2}-v_{2}-u_{2} w_{2}\right] \\
& \frac{d w_{2}}{d t}=\tau\left[-b w_{2}+u_{2} v_{2}\right]
\end{aligned}
$$

The coupling term in eq. $(5 \mathrm{~d}), \varepsilon\left(u_{1}-u_{2}\right)$, is designed by using the unity gain difference amplifier $\mathrm{U} 11$ in the circuit. The notation $\tau=1 / C_{1} R_{5}$ is the circuit time scale and can easily be adjusted by changing the values of the six capacitors $C_{1}$ to $C_{6}$ by a common factor. Thus the circuit dynamics can be made slow or fast by changing the values of the six capacitors. All component values are kept fixed except $R_{3}, R_{10}$ and $R_{17}$. The value of resistance $R_{3}$ is chosen as $34.1 \mathrm{k} \Omega$ ?when the driver generates the typical butterfly type attractor and the resistance $R_{10}$ is set at $494 \mathrm{k} \Omega$ to push the response oscillator at a stable equilibrium in an uncoupled state. Given these choices of $\mathrm{R}_{3}, \mathrm{R}_{10}$ and other components as noted in the circuit diagram, the parameters $\sigma, b$ and $r_{1}, r_{2}$ for the two coupled Lorenz oscillators are estimated as $\sigma=R_{5} / R_{1}=R_{12} / R_{8}=10$, $b=R_{5} / R_{7}=R_{12} / R_{14}=2.67$, and $r_{1}=R_{5} / R_{3}=29.33$ for the driver oscillator and $\mathfrak{k}=\mathrm{R}_{12} / \mathrm{R}_{10}=2.02$ for the response oscillator. As we increase $\mathrm{R}_{17}$ from $98 \mathrm{k} \Omega$ to $437.8 \mathrm{k} \Omega$, the coupling strength decreases and the multiscrolls $(\mathrm{n}=3,4,5,6$-scroll) gradually appear one after another. The experimental pictures of the 2D projected multiscroll attractors are shown in Fig.10 as taken from a 4channel digital oscilloscope (Tektronix, TDS 3014B, 100MHz) of a 10k data record length and maximum sampling rate $1.25 \mathrm{GS} / \mathrm{s}$. The $2 \mathrm{D}$ projections are obtained using XY plots of the oscilloscope and by monitoring the outputs of analog devices U6 and U7. They perfectly match the numerical phase portraits of n=3-, 4-, 5-, 6-scrolls in Fig.5(e)-(h) respectively. 


\section{Conclusion}

In synchronization studies, in general, interaction of oscillatory systems are usually investigated using either unidirectional, diffusive or delay coupling. However, interest on the interaction of oscillatory and resting systems have grown in recent times in the context of neuronal behaviors. We have focused here on the latter context and investigated a unidirectional coupling scheme where a double scroll type chaotic oscillator is forced into another similar type of oscillator in a resting state. By forcing the double scroll chaos into another similar oscillator in resting state and then by decreasing the coupling strength, we find interesting multiscroll dynamics in the driven system. We have applied this coupling scheme to three different systems, namely, the Chua oscillator, a modified Chua oscillator and the Lorenz system. In the case of the coupled Chua oscillator, the response oscillator shows a 4-scroll for moderately weak coupling when the trajectory jumps intermittently from one double scroll to another symmetric double scroll. This is the conventional intermittency regime as reported [Kapitaniak and Chua,1994; Suykens and Chua, 1997] earlier. However, they observed such an intermittency regime of 4-scroll in coupled selfoscillatory Chua oscillators and with additional breakpoints in its piecewise linear function. We have also derived a new 3scroll attractor by modifying the piecewise linear function in the Chua oscillator. The coupled modified Chua oscillator shows an intermittency regime of a 6 -scroll attractor. We conclude that one can always generate $2 \mathrm{n}$-scroll using our coupling scheme in the intermittency regime of a reflection symmetric type n-scroll system. No additional breakpoint is necessary which reduces the circuit comple xity. We have extended this coupling scheme to the Lorenz system, when we find even more interesting multiscroll dynamics in the weaker coupling regime. We have observed multiscroll $(\mathrm{n}=3-, 4,5-6$-scroll $)$ in the coupled Lorenz system where the dynamics appears as if unfolding one scroll after another with decreasing coupling strength. We have provided numerical as well as experimental evidences of multiscroll in both Chua oscillator and Lorenz system. We have explained the origin of the multiscroll dynamics using eigenvalue analysis. We have also presented a schematic diagram of the multiscroll trajectories in the coupled Lorenz system to elucidate their evolution in the context of stability of the equilibrium points. We have derived a numerical bifurcation diagram to find the ranges of coupling where the multiscroll is observable in the coupled Lorenz system. It is noteworthy that, in the resting state, both symmetric saddle foci of the response system (Chua oscillator or Lorenz system) are stable by a suitable choice of the parameters. However, we have forced the double scroll chaos only to one of the two saddle foci of the response system. Hence bistability must exist in the multiscroll structure corresponding to two stable foci of all systems considered here. The question of bistability is not elaborated here and left for future studies.

\section{Acknowledgements}

S.K.D. and P.K.R acknowledge partial support by the DST, India under the Gant \#SR/S2/HEP -03/2005. S.K.D also acknowledges the hospitality of the National Center for Theoretical Sciences in Taiwan, where part of this work was done. C.K.H. was supported by the National Science Council in Taiwan under Grant \# NSC 95-2112-M001-008 and National Center for Theoretical Sciences in Taiwan. J.K acknowledges the joint support of the Humboldt Foundation, Germany and CSIR, India by the Humboldt-CSIR Reciprocity Research Award.

\section{References}

Arena P., Baglio S., Fortuna L.and Manganaro G., [1996] "Generation of n-double scrolls via cellular neural networks," Int.J.Circuit Theory Application., 24 (3), 241-252.

Cafagna D. and Grassi G., [2003] "New 3-D-scroll attractors in hyperchaotic Chua's circuit forming ring," Int.J.Bifur.Chaos, 13 (10), 2889-2903.

Chua L.O., Komuro M., Matsumoto T., [1986] “Double scroll family, IEEE Trans.Cir.Systs., 33 (11), 1072-1118 (1986).

Dana S.K., Blasius B. and Kurths J. [2006] "Experimental evidence of anomalous phase synchronization in two diffusively coupled Chua oscillators”, CHAOS, 16, 023111.

Kapitaniak T., Chua L.O.and Zhong G.-Q., [1990] "Experimental hyperchaos in coupled Chua's circuit," IEEE Trans. Cir.Systs.-I, 41 (7), 499-503.

Kapitaniak T., Chua L.O., [1994] "Hyperchaotic attractors of unidirectionally-coupled Chua's circuit," Int. J. Bifur Chaos, 4 (2), 477- 482

Kennedy M.P., [1993], “Three steps to chaos. II. A Chua's circuit primer”, IEEE Trans. Circuits Syst., I: 40 (10), $657-674$. 
Lü J., Yu X. and Chen G., [2003] "Generating chaotic attractors with multiple merged basin: A switching piecewise linear control approach,” IEEE Trans.Cir.Systs.-I, 50, 198-207.

Lii J., Chen G., [2006] “Generating multiscroll chaotic attractors: Theories, Methods and Applications,” Int. J.Bifur Chaos, 16 (4), 775-858.

Lü J., Chen G., Hu X., Leung H., [2004] "Design and analysis of multiscroll chaotic attractors from saturated function series," IEEE Trans Cir.Systs.-I, 51 (12), 2476-2490.

Lü J., Han F., Yu X., Chen G., [2004a] "Generating 3-D multiscroll chaotic attractors: A hysteresis series switching method," Automatica, 40 (11), 1677-1877.

Lü J. , Yu S., Leung H. and Chen G., [2006] "Experimental verification of multidirectional multiscroll chaotic attractors," IEEE Trans. Cir.Systs.-I, 53 (1), 149-165.

Osipov G., Kurths J., Zhou C. S. [2007], Synchronization in oscillatory networks, Springer Complexity, Berlin.

Ozoguz S., Alwakil A.S. and Salama K.N., [2002] "n-scroll chaos generator using nonlinear transconductor," Electron.Lett., 38, 685-686.

Pecora L. and Carroll T., Nonlinear Dynamics in Circuits, World Scientific, Singapore, 1995

Pikovsky A.S., Rosenmblum, M., Kurths J. [2000] "Phase synchronization in regular and chaotic systems", Int. J. Bifur. Chaos, 10(1), 2291-2306.

Pikovsky A., Rosenblum M., and Kurths J. [2001], Synchronization: A Concept in Nonlinear Sciences, Cambridge University Press, Cambridge, MA.

Roy P.K., Chakraborty S., Dana S.K., [2003] "Experimental observations on the effect of coupling on different synchronization phenomena in two coupled nonidentical Chua's oscillators," Chaos, 13 (1), 342-355.

Singh B.K. and Hu C.-K.,[2005] "Self-feedback chaos control for nonlinear systems,” (preprint).

Suykens J.A.K. and Vandewalle J.,[1993] “Generation of n-double scrolls (n=1,2,3,4,..)," IEEE Trans. Cir.Systs.-I, 40 (7), 861-867.

Suykens J.A.K. and Chua L.O., [2001] "n-double scroll hypercubes in 1-D CNNs," Int.J.Bifur.Chaos, 7(8), 1873-1885.

Tang W.K.S., Zhong G.Q., Chen G. and Man K.F., [2001] “Generation of n-scroll attractors via sine function,” IEEE Trans.Cir. Systs.-I, 48, 1369-1372.

Yalcin M.E., Suykens J.A.K. and Vandewalle J., [2000] "Experimental confirmation of 3- and 5-scroll attractors from a generalized Chua's circuit," IEEE Trans.Cir.Systs.-I, 47 (3), 425-429.

Yalcin M.E., Ozoguz S., Suykens J.A.K. and Vandewalle J., [2001] “n-scroll chaos generator: a simple circuit model," Electron.Lett., 37 (3), 147-148.

Yu S., Lu J., Leung H., Chen G., [2005] "Design and implementation of n-scroll chaotic attractors from a general Jerk Circuit," IEEE Trans.Cir.Systs. -I, 52 (7), 1459-1476.

Yu S., Lü J., W.K.S.Tang and G.Chen, [2006] "A general multiscroll Lorenz system family and its realization via digital signal processors," Chaos, 16, 033126. 